\title{
Analysis of the carbon-rich very metal-poor dwarf G77-61*
}

\author{
B. Plez ${ }^{1}$ and J. G. Cohen ${ }^{2}$ \\ 1 GRAAL, CNRS UMR 5024, Université Montpellier II, 34095 Montpellier Cedex 5, France \\ e-mail: plez@graal .univ-montp2 .fr \\ 2 Palomar Observatory, Mail Stop 105-24, California Institute of Technology, Pasadena, Ca 91125, USA
}

Received 28 September 2004 / Accepted 5 January 2005

\begin{abstract}
We present an analysis of the carbon dwarf G77-61, a known binary, based on high resolution Keck spectra. G77-61 has a very-low metallicity, although not as extreme as what was previously conjectured. This star is very carbon-enhanced, the spectra showing very strong $\mathrm{CH}, \mathrm{CN}$, and $\mathrm{C}_{2}$ bands of both ${ }^{13} \mathrm{C}$ and ${ }^{12} \mathrm{C}$ isotopes. Atomic lines are sparse, and often blended, but we were able to derive abundances for $\mathrm{Na}, \mathrm{Mg}, \mathrm{Ca}, \mathrm{Cr}, \mathrm{Mn}$, and $\mathrm{Fe}$. Its $[\mathrm{Fe} / \mathrm{H}]$ of $-4.03 \pm 0.1$ places it among the lowest metallicity stars known, and its very high $[\mathrm{C} / \mathrm{Fe}]=+2.6$, and $[\mathrm{N} / \mathrm{Fe}]=+2.6$ among the most $\mathrm{C}$ and N-rich metal-poor stars. The carbon isotopic ratio is ${ }^{12} \mathrm{C} /{ }^{13} \mathrm{C}=5 \pm 1$. No overabundance of s- or r-process elements is detectable, but the upper limits that can be set on these overabundances are not very constraining.
\end{abstract}

Key words. stars: abundances - stars: carbon - stars: Population II - stars: individual: G77-61

\section{Introduction}

The star G77-61 was first identified as a nearby carbon dwarf by Dahn et al. (1977). They reanalyzed its previous parallax determination by Routly (1972) to find $M_{V}=+9.6 \pm 0.6 \mathrm{mag}$. Dearborn et al. (1986) established that G77-61 is a binary with an unseen companion and with a period of $\sim 245$ days, suggesting a cool white dwarf of much higher mass than the visible object as the second star. The primary was later spectroscopically analysed by Gass et al. (1988), who concluded that its metallicity was extremely low, $[\mathrm{Fe} / \mathrm{H}]=-5.5 \mathrm{dex}$ (on our adopted scale with $\log A(\mathrm{Fe})=7.45$ ). No other spectroscopic investigation has been carried out since then.

The discovery of a large number of very metal-poor, carbon-rich objects, with diverse additional peculiarities, particularly $r$-process or/and $s$-process enrichment, and the discovery of the most iron-poor star known, HE0107-5240 (Christlieb et al. 2002), at $[\mathrm{Fe} / \mathrm{H}]=-5.3$, which is also very $\mathrm{C}$-rich, all contribute to a renewed interest in the star G77-61.

\section{Observations}

The star G77-61 was observed several times using HIRES (Vogt et al. 1994) at the Keck Observatory. The first set of spectra (3 exposures, each $1200 \mathrm{~s}$ long) were obtained on the night of Sep. 29, 2002. They covered a spectral range from 3780 to $5320 \AA$, at a resolution of 34000 with small gaps between the

^ Based in part on observations obtained at the W. M. Keck Observatory, which is operated jointly by the California Institute of Technology, the University of California, and the National Aeronautics and Space Administration. orders at the red end of the range. A 1.1 arcsec wide slit was used, and a signal to noise ratio (SNR) of 100/spectral resolution element ( 4 pixels wide) in the continuum in the middle of this spectral range was achieved. This was calculated assuming Poisson statistics and ignoring issues of cosmic ray removal, flattening etc. The star was re-observed August 19, 2003 to cover the region from 5000 to $7900 \AA$, with a resolution of 34000 . Three separate exposures were required for full spectral coverage. On line signal calculations used the order just blueward of the center of the CCD at a wavelength of $5865 \mathrm{~A}$. Much shorter exposure times were required to achieve the desired SNR of 75 to $100 /$ spectral resolution element as the star is so red.

These spectra were reduced using a combination of Figaro scripts (Shortridge 1993) and the software package MAKEE ${ }^{1}$. Normal procedures were followed until the final step, which involves fitting a low order polynomial to the continuum to remove the last residuals of the variation of the instrumental response with wavelength. Since the continuum is essentially impossible to determine in this heavily banded star, even at this spectral resolution, this step was omitted. Typical residuals removed at this stage are about $10 \%$ of the mean continuum within an order and are locally smooth.

The observed heliocentric radial velocity for G77-61 is $-29 \mathrm{~km} \mathrm{~s}^{-1}$ on September 29, 2002, and $-20 \mathrm{~km} \mathrm{~s}^{-1}$ on August 19, 2003, with somewhat large uncertainty due to

${ }^{1}$ MAKEE was developed by T. A. Barlow specifically for reduction of Keck HIRES data. It is freely available on the world wide web at the Keck Observatory home page,

http://www2 . keck . hawaii . edu:3636/. 
the strong veiling. These values fit very well with those predicted from the orbit of Dearborn et al. (1986): -29.6 and $-18.5 \mathrm{~km} \mathrm{~s}^{-1}$.

\section{Analysis}

The spectra were analysed through spectrum synthesis, the very strong veiling by molecular bands not allowing equivalent width determinations.

\subsection{Stellar parameters}

Our $B-V$ photometry from the Swope 1-m telescope at the Las Campanas Observatory, (Ivans \& Cohen, private communication), $B=15.70$ and $V=13.97$, gives $B-V=1.73 \mathrm{mag}$. Dahn et al. (1977) provide $V=13.90, B-V=1.70$. The trigonometric parallax of $16.9 \pm 2.2$ mas (Harris et al. 1998), and the $V$ magnitude lead to $M_{V}=10.1 \pm 0.27$, assuming no reddening, as the star is nearby. Schlegel et al.'s extinction maps (1998) give $E(B-V)=0.100$ at this position. The Galactic latitude of G77-61 is $-41.51^{\circ}$, corresponding to a height of $39 \mathrm{pc}$ above the Galactic plane, resulting in about $25 \%$ of the total extinction, or $0.025 \mathrm{mag}$, well below all other uncertainties. The IR colours $J-H=0.684, H-K=0.337$, and $J-K=1.007$ in the Johnson system (Bessell \& Brett 1988) are obtained from 2MASS photometry (Skrutskie et al. 1997, and Cutri et al. 2003) $J=11.470$ (0.022), $H=10.844$ (0.024), $K=10.480$ (0.019), using the relations of Carpenter (2001). The $K$ magnitude is 10.52 in the Johnson system, which transforms to an absolute $M_{K}=6.62 \pm 0.27$. Carbon-rich dwarf MARCS models around $T_{\text {eff }}=4000 \mathrm{~K}$ and $[\mathrm{Fe} / \mathrm{H}]=-3$ or -4 have bolometric corrections $\mathrm{BC}_{K} \approx 2.25$, and $\mathrm{BC}_{V} \approx-0.65$. Thus, the absolute bolometric magnitude is $M_{\text {bol }} \approx 9.45$ from $V$, and 8.87 from $K$. Dearborn et al. (1986) find $\mathrm{BC}_{V}=-0.96$ from spectrophotometric observations, leading to $M_{\mathrm{bol}}=9.14$. We adopt the average of the $K$ and $V$ values, $M_{\text {bol }}=9.16$ (i.e. $L=0.017 L_{\odot}$ ).

We adopt $T_{\text {eff }}=4000 \pm 200 \mathrm{~K}$, obtained from interpolating the observed $V-J$ and $V-K$ colors in the predicted color grid of Houdashelt et al. (2000). We assume that the star is sufficiently nearby that the reddening is negligible. The uncertainty in $T_{\text {eff }}$ is not due to the observational photometric errors but rather to the modeling uncertainties. The Houdashelt et al. (2000) color grid was calculated assuming scaled Solar compositions extending to $[\mathrm{Fe} / \mathrm{H}]$ as low as -3 dex, but $\mathrm{G} 77-61$ has a highly anomalous composition with very large $\mathrm{C}$ excess. The $\mathrm{C}_{2}$ bands reduce the flux integrated over the $V$ bandpass below that of the true continuum by $\sim 35 \%$. The effect of the prominent $\mathrm{C}_{2}$ absorption, which is not included in the models at all, has been compensated for in an approximate way, but still leaves a large uncertainty in $T_{\text {eff. }}$ The range of $T_{\text {eff }}$ from the three previous analyses of this star listed in Sect. 1 is 4000 to $4200 \mathrm{~K}$.

For $T_{\text {eff }}=4000 \mathrm{~K}$ we deduce that $R=0.27 R_{\odot}$, and $\log g=$ 5.27 assuming $M=0.5 M_{\odot}$, assuming $M=0.3 M_{\odot}$ leads to $\log g=5.05$.

While evolutionary tracks for metal poor stars with such extreme $\mathrm{C}$ enhancement are not available, the new Yale-Yonsei isochrones (Kim et al. 2002; Yi et al. 2003) with $\alpha$-enhancement give an indication of whether these values are in accord with those expected for an old lower main sequence star. We examine the sequence of metal poor isochrones with $\alpha$-enhancements of a factor of four and age of $9 \mathrm{Gyr}$. The inferred luminosity of G77-61 then corresponds to a mass of $0.38 M_{\odot}$. The $T_{\text {eff }}$ of the isochrone at this luminosity, which is probably quite uncertain, is $\sim 300 \mathrm{~K}$ lower than the value we adopt.

\subsection{Model atmospheres and line data}

Using the estimates of $T_{\text {eff }}$, and $\log g$, models were calculated with the MARCS code (Gustafsson et al. 1975; Plez et al. 1992; Edvardsson et al. 1993; Asplund et al. 1997; Gustafsson et al. 2003), at metallicities between -5 and -3 , for various $\mathrm{C} / \mathrm{H}$ ratios. The synthetic spectra were computed with the TURBOSPECTRUM package (Alvarez \& Plez 1998), using atomic lines from the VALD database (Kupka et al. 1999). The data for the lines we use in the abundance analysis is listed by Hill et al. (2002), and Cayrel et al. (2004). The molecular line lists for $\mathrm{CH}$, and $\mathrm{CN}$ were specially assembled. These lists, used by Hill et al. (2002), were further updated. The $\mathrm{CH}$ line list was improved by shifting a number of line positions by $0.1 \AA$ or less between 4200 and $4275 \AA$, for ${ }^{13} \mathrm{CH}$ A-X lines, and to a lesser extent ${ }^{12} \mathrm{CH}$ lines (using in particular Richter \& Tonner 1967 , as well as a Keck spectrum of the carbon-rich metal-poor star HE0212-0557). The $\mathrm{C}_{2}$ line list was taken from Querci et al. (1971), and updated by scaling the $g f$-values to agree with modern lifetime and intensity measurements (Cooper \& Nicholls 1975; Grevesse et al. 1991; Erman \& Iwamae 1995; Langhoff et al. 1990). The situation is far from satisfactory for spectrum synthesis with this latter list, and efforts are under way to improve the situation. We did not use $\mathrm{C}_{2}$ lines to derive the $\mathrm{C}$ abundance, and avoided the use of other lines situated within $\mathrm{C}_{2}$ bands, where a risk of blending exists.

It is important to use a model computed with abundances close to the spectroscopic determination. Figure 1 shows the thermal and pressure structure of the final model adopted, with abundances from Table 1, together with a second model with solar abundances uniformly scaled down by -4 dex and with $[\alpha / \mathrm{Fe}]=+0.4$, and a third model with in addition CNO abundances set to their final values. The thermal structure of the model is affected by the abundance of species that contribute importantly to the opacity (mostly $\mathrm{C}$ and $\mathrm{N}$, due to the predominance of $\mathrm{C}_{2}$ and $\mathrm{CN}$ in the spectrum), and less importantly by the abundance of electron donors $(\mathrm{Mg}, \mathrm{Na}$, and Ca) which has a impact on the $T-P_{\mathrm{e}}-P_{\text {gas }}$ relation in the outermost layers. This is further illustrated in the spectrum synthesis. Changing from a model with scaled solar abundances $([\mathrm{X} / \mathrm{H}]=-4.0)$, and $[\alpha / \mathrm{Fe}]=+0.4$, to a model with the abundances of Table 1 results for example in a change of more than $0.3 \mathrm{dex}$ in the determination of the abundance of $\mathrm{Na}$ from the Na I D line. This is illustrated in Fig. 2 where synthetic spectra computed with various $\mathrm{Na}$ abundances in the three model atmospheres are compared to the observation. In the case of the two models with equal abundances of $\mathrm{C}, \mathrm{N}$, and only differences in some elements (esp. $\mathrm{Na}, \mathrm{Mg}, \mathrm{Ca}$ ), the differences are due to the impact of the electron donors in the line 


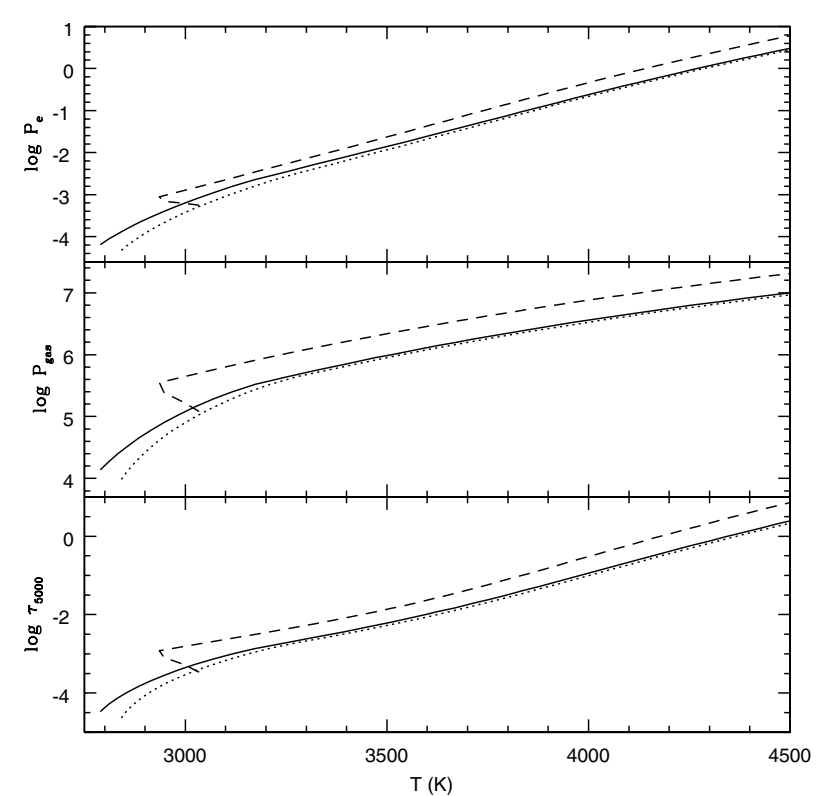

Fig. 1. Effect of abundance changes on model atmosphere thermal and pressure structure. Full line is the final model, with the abundances of Table 1, dashed line is a model with solar abundances scaled by $-4 \mathrm{dex}$, and $[\alpha / \mathrm{Fe}]=0.4$, dotted line has in addition $\mathrm{CNO}$ abundances to their final value.

Table 1. Abundances derived for G77-61.

\begin{tabular}{llllll}
\hline \hline Element & $\log \epsilon(\mathrm{X})$ & Error & {$[\mathrm{X} / \mathrm{H}]$} & {$[\mathrm{X} / \mathrm{Fe}]$} & Wavelength $(\AA)$ \\
\hline $\mathrm{C}(\mathrm{CH})^{*}$ & 7.0 & \pm 0.1 & -1.4 & +2.6 & \\
$\mathrm{~N}(\mathrm{CN})$ & 6.4 & \pm 0.1 & -1.4 & +2.6 & \\
$\mathrm{Li} \mathrm{I}$ & $<1$ & & $<0$ & $<4$ & 6708 \\
$\mathrm{Na}$ I & 2.9 & \pm 0.2 & -3.4 & +0.60 & 5889 \\
$\mathrm{Mg}$ I & 4.0 & \pm 0.2 & -3.5 & +0.49 & 5183,5172 \\
$\mathrm{~K} \mathrm{I}{ }^{b}$ & 2.3 & \pm 0.3 & -2.8 & +1.21 & 7665,7699 \\
$\mathrm{Ca}$ I & 2.7 & \pm 0.2 & -3.7 & +0.37 & 4226 \\
$\mathrm{Ca}$ II & 2.9 & \pm 0.2 & -3.5 & +0.57 & $8498,8542,8662$ \\
$\mathrm{Cr}$ I & 2.0 & \pm 0.2 & -3.7 & +0.36 & 5206,5208 \\
$\mathrm{Mn} \mathrm{I}$ & 1.3 & \pm 0.3 & -4.1 & -0.06 & 4030 \\
$\mathrm{Fe} \mathrm{I}$ & 3.42 & \pm 0.15 & -4.03 & & $3887,3895,3899$ \\
& & & & & $4045,4063,4071$ \\
$\mathrm{Zn} \mathrm{I}$ & & & & $<2.7$ & 4810 \\
$\mathrm{Sr} \mathrm{II}$ & & & & $<0$ & 4077 \\
$\mathrm{Ba}$ II & & & & $<1$ & 4554,4934 \\
$\mathrm{Eu} \mathrm{II}$ & & & & $<3 ?$ & 4129,4205 \\
\hline$a{ }^{12} \mathrm{C} /{ }^{13} \mathrm{C}=5 \pm 1$. & & & & \\
Th & & & &
\end{tabular}

${ }^{b}$ The $\mathrm{K}$ abundance is quite uncertain: blend with $\mathrm{CN}$.

forming layers. Their resulting difference is about 0.1 dex in the determination of the $\mathrm{Na}$ abundance. Interestingly, deeper in the atmosphere, around $\tau_{\text {Ross }}=1$, about $80 \%$ of the electrons arise from $\mathrm{H}_{3}^{+}$. The obvious impact this has on $\mathrm{H}^{-}$opacity demonstrates the importance of including $\mathrm{H}_{3}^{+}$in the molecular equilibrium, as recently shown for the evolution of zero-metal stars by Harris et al. (2004). We use the partition function of Neale \& Tennyson (1995) for $\mathrm{H}_{3}^{+}$.

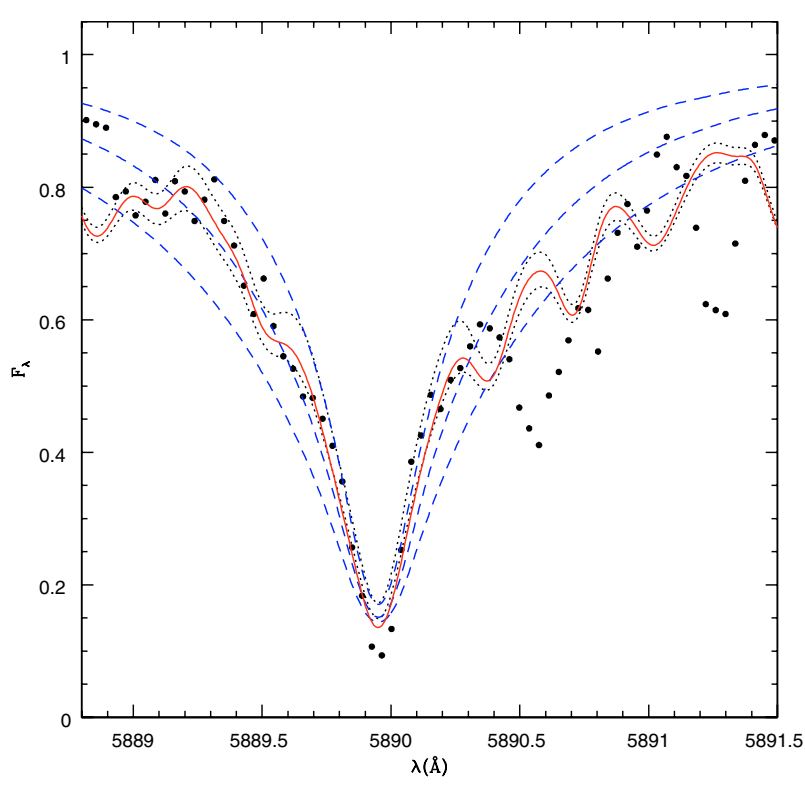

Fig. 2. Effect of model atmosphere changes on the Na I D line. Dots are observations; full line is the synthetic spectrum from the model atmosphere with the final abundances, including $\log A(\mathrm{Na})=2.92$; dotted lines are for $2 \mathrm{Na}$ abundances: 2.92 and 3.22, and a model with solar abundances scaled by -4 dex, except $\mathrm{CNO}$ which have their final value, and $[\alpha / \mathrm{Fe}]=0.4$ (dotted line model of Fig. 1); finally, dashed lines are for $\mathrm{Na}$ abundances of 2.32, 2.62 and 2.92 and the model with solar abundances scaled by -4 dex (dashed line model of Fig. 1). The features in the observed spectrum that are not matched by our calculations are due to $C_{2}$.

The effect of changes in $\mathrm{C}$ and $\mathrm{N}$ abundances on the emergent spectrum is even more dramatic, through the change in blanketing of $\mathrm{C}_{2}, \mathrm{CN}$ and $\mathrm{CH}$, which affects the thermal structure of the model (see Figs. 1 and 2). The $\mathrm{O}$ abundance, which we cannot determine, has no impact as long as it stays well below the C abundance. Low-resolution IR spectra (Joyce 1998) as well as IR photometry (Dahn et al. 1977) show very faint $\mathrm{CO}$ bands, in favor of a low oxygen abundance. We adopted here $\log A(\mathrm{O})=5.0$.

\subsection{Results}

Abundances were first derived for $\mathrm{C}$ using the $\mathrm{CH} A-X$ and $B-X$ bands around 3890 and $4100-4200 \AA$ (see Fig. 3), with checks on the $\mathrm{C}_{2}$ Swan bandheads at $5160 \AA$ and $4735 \AA$. The $\mathrm{N}$ abundance was estimated using the $\mathrm{CN} A-X$ red bands around $8000 \AA$. Note that an increase of the derived carbon abundance would lead to a decrease of the nitrogen abundance by the same amount. The $\mathrm{C}_{2}$ Swan and Phillips bands, although very strong and displaying obvious bandheads for the ${ }^{12} \mathrm{C}_{2}$, the ${ }^{12} \mathrm{C}^{13} \mathrm{C}$, and ${ }^{13} \mathrm{C}_{2}$ isotopic combinations, could not be used, because of the absence of reliable line list. The ${ }^{12} \mathrm{C} /{ }^{13} \mathrm{C}$ isotopic ratio of $5 \pm 1$, was derived using $18{ }^{13} \mathrm{CN}$ lines between 7925 and $7966 \AA$. Wavelengths of ${ }^{13} \mathrm{CN}$ lines from the list of Plez were shifted by $0.1 \AA$ to improve the match to the observations. Figures 4 and 5 show parts of these regions. The synthetic spectrum without $\mathrm{CN}$ lines included is also plotted. Faint lines appear in this synthetic spectrum, which seem well placed 


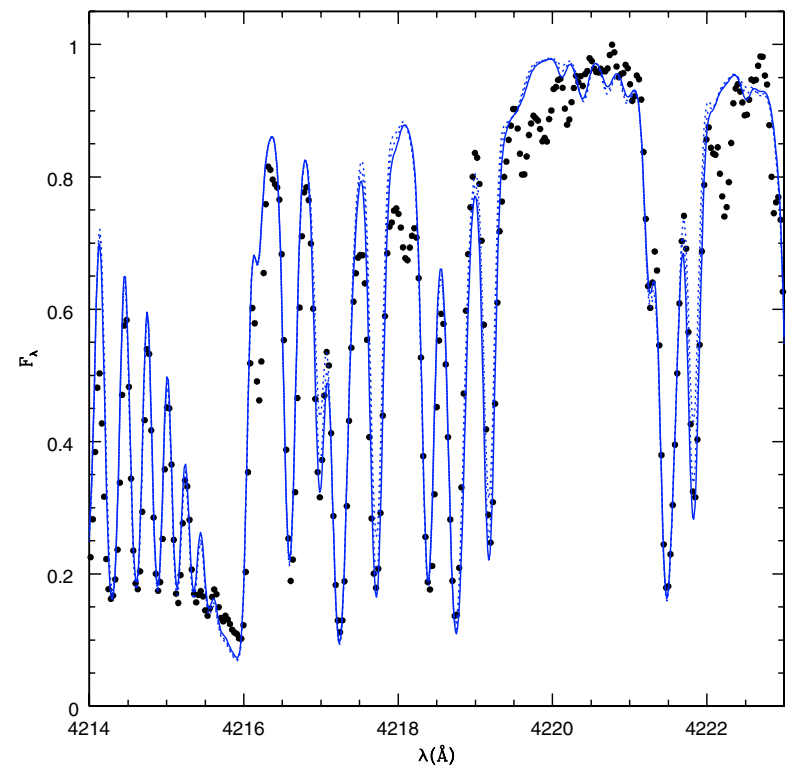

Fig. 3. $\mathrm{CH} A-X$ lines and $\mathrm{CN} B-X$ bandhead. Dots are the observed spectrum. Synthetic spectra were calculated for ${ }^{12} \mathrm{C} /{ }^{13} \mathrm{C}=5$ (final adopted value), 9, and 20. All abundances have their final value.

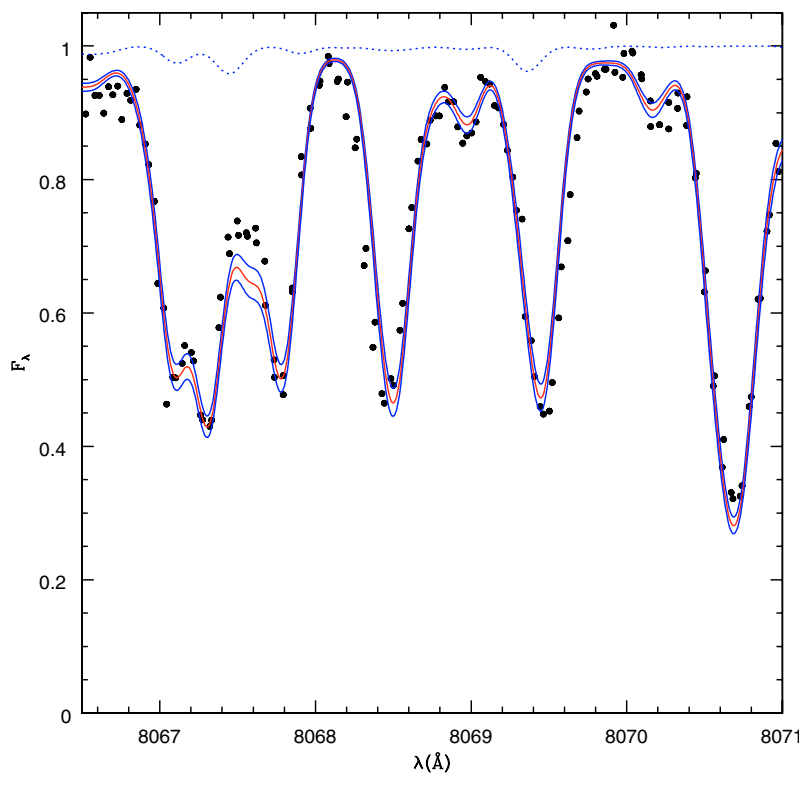

Fig. 4. CN red system lines. Dots are two observed spectra, with different setups. Synthetic spectra were calculated for 3 different $\mathrm{N}$ abundances, $\log A(\mathrm{~N})=6.32,6.42,6.52$, and without $\mathrm{CN}$ lines.

but too faint to fit the observed spectrum. These lines are low excitation $\mathrm{C}_{2}$ lines from the Phillips bands, for which we don't have a reliable line list. Efforts to improve the situation are ongoing, as these unsaturated lines seem promising to determine the carbon abundance in stars similar to G77-61.

The impact of microturbulence is not negligible on the strong saturated lines. We could only use a handful of strong atomic lines. Most molecular lines are also somewhat saturated. We could thus not derive a microturbulence parameter from the observations. We decided to use $0.1 \mathrm{~km} \mathrm{~s}^{-1}$ for the following reason: in the Sun the ratio of microturbulence parameter to the maximum MLT velocity is $1.3 / 2.5$. The MLT velocity

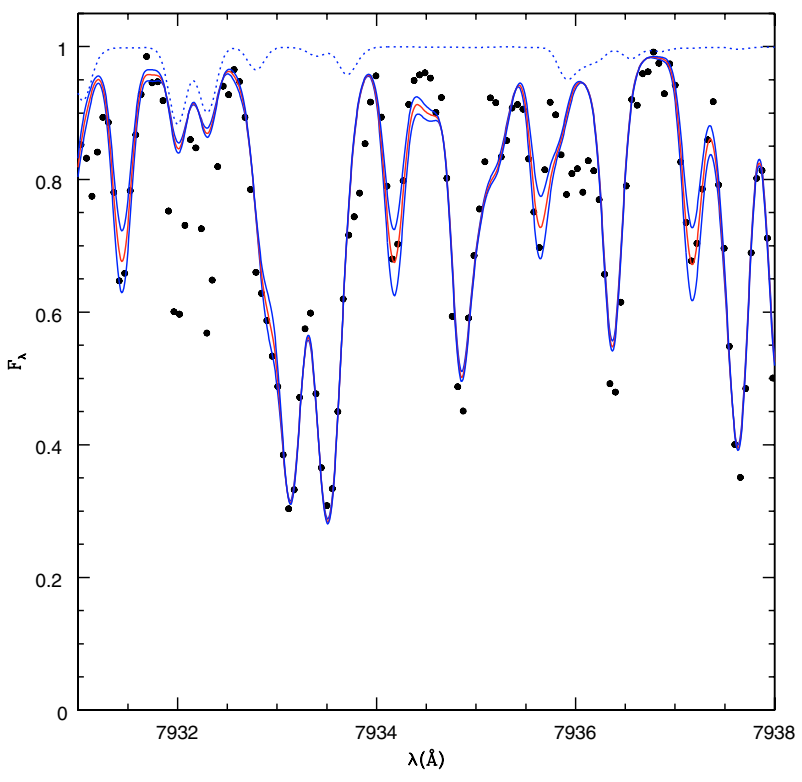

Fig. 5. Red system lines of ${ }^{12} \mathrm{CN}$ and ${ }^{13} \mathrm{CN}$. Spectra were computed for 3 different isotopic ratios: ${ }^{12} \mathrm{C} /{ }^{13} \mathrm{C}=4,6,9$, as well as without any $\mathrm{CN}$ lines. The (too) faint spectral lines appearing in the latter case (dotted line) are $\mathrm{C}_{2}$ lines from the Phillips transition system. The line list we use for $\mathrm{C}_{2}$ is not reliable.

in our $\mathrm{C}$ dwarf model is $0.22 \mathrm{~km} \mathrm{~s}^{-1}$, reached around $\tau_{5000}=$ 1. Assuming the same ratio as for the Sun, which is reasonable if we suppose that microturbulence measures convective overshooting in the upper layers of the atmosphere, we find a microturbulent velocity of the order of $100 \mathrm{~m} / \mathrm{s}$, which is one order of magnitude smaller than the thermal velocity of atoms at $3000 \mathrm{~K}$. Actually, convection reaches optically thin layers in the carbon dwarf models with velocities of about $100 \mathrm{~m} / \mathrm{s}$ at $\tau_{5000}=0.01$, which reinforce our estimate for the microturbulence parameter. Using $1 \mathrm{~km} \mathrm{~s}^{-1}$ would not impact the abundances derived here very much. Only the use of a very unlikely microturbulence parameter of the order of $2 \mathrm{~km} \mathrm{~s}^{-1}$ or more would induce significant abundance changes, and a notably worse match to the observations.

Unblended atomic lines are rare in the spectrum of G77-61, and most are strong. The abundances we present are based on few lines, often with low excitation energies, and subject to non-LTE effects. Large errors might have occurred due to an inappropriate description of the upper layers of the stellar atmosphere. We avoided lines in regions of the spectrum where the synthetic spectra did not reproduce well enough the molecular veiling. In particular, the region below $4000 \AA$ A shows numerous large absorption features, of unidentified origin. These features show up in the spectra of other cool C-rich, metal-poor stars as well, and efforts toward their identification should be made.

The abundances are presented in Table 1, where we use as reference solar abundances the values from Grevesse \& Sauval (1998), except for $\mathrm{C}=8.41, \mathrm{~N}=7.80$, and $\mathrm{O}=8.67$ which we take from Allende-Prieto et al. (2002a), Asplund (2003), and Asplund et al. (2004), and $\mathrm{Fe}=7.45$ from Asplund et al. (2000). Our solar abundances are the same as Christlieb at al. (2004) and Cohen et al. (2004). 

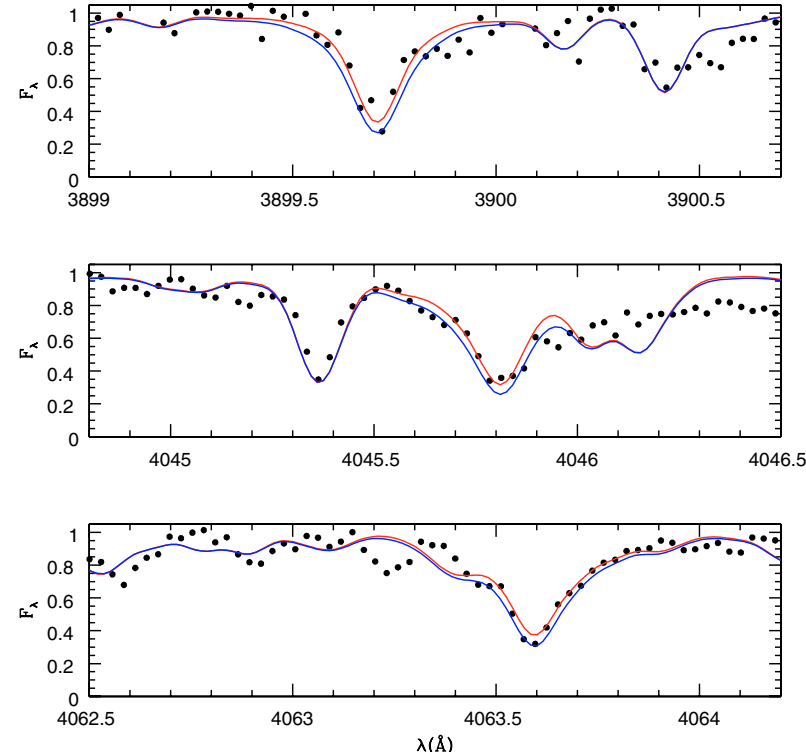

Fig. 6. Observed (dots) and calculated spectra (full lines) of $3 \mathrm{Fe} I$ lines, for $2 \mathrm{Fe}$ abundances: 3.30 and 3.60, using the final model atmosphere parameters.

The uncertainties from the quality of the fit of individual lines are of the order of 0.15 dex. In some cases (K I) possible additional blends increase the uncertainty to greater values (0.3 dex or more). For Fe I (see Fig. 6), the situation is better, as 6 lines could be used, that are not resonance lines, contrary to $\mathrm{Na}$, or $\mathrm{K}$. The standard deviation from the 6 lines is 0.1 dex. The uncertainties quoted in Table 1 are estimated on this basis. They do not include any systematic error that could arise from a change of stellar parameters. The abundances derived using the model with solar abundances scaled down by 4 dex, except the alpha elements $([\alpha / \mathrm{Fe}]=+0.4)$, and the final $\mathrm{CNO}$ abundances, differed from the abundances quoted in Table 1 by 0 dex for $\mathrm{Ca}$, by less than 0.1 dex for $\mathrm{Fe}$, by $+0.15 \mathrm{dex}$ for $\mathrm{Mg}$ and $\mathrm{Cr}$, by $0.3 \mathrm{dex}$ for $\mathrm{Mn}$, and $0.6 \mathrm{dex}$ for $\mathrm{Na}$ I. At the upper limit to the $T_{\text {eff }}$ for G77-61, i.e. $4200 \mathrm{~K}$, [Fe/H] becomes -3.85 . This is a reasonably secure upper limit to the metallicity of this star. An increase of the gravity to $\log g=5.5$ reduces the Fe abundance by 0.3 dex. For Ca II, the changes are -0.1 dex when the gravity is increased to $\log g=5.5$, and $+0.3 \mathrm{dex}$ for an increase of $T_{\text {eff }}$ to $4200 \mathrm{~K}$, while the $\mathrm{Na} \mathrm{I}$ abundance rises by $+0.5 \mathrm{dex}$. These values give an estimate of the systematic errors that may affect our results, not accounting for possible non-LTE effects.

\section{Discussion}

We have shown that G77-61 is an extremely metal poor dwarf with $[\mathrm{Fe} / \mathrm{H}]-4.03$ dex which is highly $\mathrm{C}$-enhanced, having $N(\mathrm{C}) / N(\mathrm{Fe}) 500$ times the Solar ratio, so that $[\mathrm{C} / \mathrm{H}]$ is -1.4 dex. There are only $\sim 10$ stars known in the Galaxy with $[\mathrm{Fe} / \mathrm{H}<$ -3.5 dex verified by a detailed abundance analysis ${ }^{2}$. G77-61 is arguably the third most Fe-poor star known in the Galaxy

2 These are CD-38 245 , CS 22162-002, CS 22885-096 and CS 22949-037, studied in detail by Norris et al. (2001), CS 22172-002, CS 22968-014 and BS 16467-062 from the sample of Cayrel et al. (2004), CS 29498-043 Aoki et al. (2002a,b, 2004),
Table 2. Comparison of G77-61 with normal extremely metal poor stars.

\begin{tabular}{lllll}
\hline \hline & $\begin{array}{l}\text { Sun } \\
\text { Element }\end{array}$ & $\begin{array}{l}\text { G77-61 } \\
\log (\mathrm{X})\end{array}$ & $\begin{array}{l}\text { Mean dwarfs }^{a} \text { (Fe] } \\
{[\mathrm{X} / \mathrm{Fe}]}\end{array}$ & $\begin{array}{l}\text { Mean giants }^{b} \\
{[\mathrm{X} / \mathrm{Fe}]}\end{array}$ \\
\hline $\mathrm{C}(\mathrm{CH})$ & 8.39 & +2.6 & & \\
$\mathrm{~N}(\mathrm{CN})$ & 7.80 & +2.6 & & \\
$\mathrm{Li} \mathrm{I}$ & 1.10 & $<4$ & & -0.24 \\
$\mathrm{Na}$ I & 6.33 & +0.60 & & +0.23 \\
$\mathrm{Mg}$ I & 7.54 & +0.49 & +0.52 & +0.27 \\
$\mathrm{~K} \mathrm{I}$ & 5.12 & +1.21 & & +0.22 \\
$\mathrm{Ca}$ I & 6.36 & +0.37 & +0.31 & \\
$\mathrm{Ca}$ II & 6.36 & +0.57 & & -0.51 \\
$\mathrm{Cr}$ I & 5.67 & +0.36 & -0.21 & $-0.52^{c}$ \\
$\mathrm{Mn}$ I & 5.39 & -0.06 & -0.55 & $-3.01^{e}$ \\
$\mathrm{Fe}$ I & 7.45 & $-4.03^{d}$ & $-2.7^{e}$ & +0.48 \\
$\mathrm{Zn} \mathrm{I}$ & 4.60 & $<2.7$ & +0.52 & \\
$\mathrm{Sr}$ II & 2.97 & $<0$ & $-0.44^{f}$ & \\
$\mathrm{Ba}$ II & 2.13 & $<1$ & -0.32 & \\
Eu II & 0.51 & $<3$ & & \\
\hline
\end{tabular}

${ }^{a}$ Sample of Cohen et al. (2004).

${ }^{b}$ Sample of Cayrel et al. (2004), from their regression at $[\mathrm{Fe} / \mathrm{H}]=$ -4.0 .

${ }^{c}$ The NLTE 0.4 dex correction Cayrel et al. uses has been removed.

${ }^{d}[\mathrm{Fe} / \mathrm{H}]$.

${ }^{e}$ Median $[\mathrm{Fe} / \mathrm{H}]$ of the sample.

${ }^{f}$ Corrected for difference of reference solar $\mathrm{Sr}$.

today; only HE $0107-5240$ is significantly more metal poor. It is interesting that many of these stars are also extremely C-enhanced ${ }^{3}$; HE 0107-5240 would appear as a C-star, as does G77-61, were it a cool dwarf rather than a subgiant with a much hotter $T_{\text {eff. }}$ Furthermore, G77-61 is the only star in this group which was originally noticed through its high proper motion; all the others were found in spectroscopic surveys.

Results from large recent surveys of very metal poor stars (Cayrel et al. 2004, for giants; and Cohen et al. 2004, for dwarfs) have now been published. Cohen et al. (2004) have pointed out that the abundance ratios $[\mathrm{X} / \mathrm{Fe}]$ for elements between $\mathrm{Mg}$ and $\mathrm{Zn}$, while they may be functions of $[\mathrm{Fe} / \mathrm{H}]$, are constant to within the observational error at a fixed value of $[\mathrm{Fe} / \mathrm{H}]$. They also provide evidence that this is true of most $\mathrm{C}$-rich stars, ignoring the CNO elements of course. Because of the complex spectrum of G77-61 with its many strong molecular bands including $\mathrm{C}_{2}$, we could only determine abundances for a limited set of elements. These are compared with the mean abundances derived for very metal poor giants and for dwarfs with normal CNO from Cayrel et al. (2004) and Cohen et al. (2004) in Table 2. To within the errors, G77-61 appears to also show similar abundance ratios, apart from the

BS 16545-0089 and HE 0218-2738 from the sample of Cohen et al. (2004), and HE 0107-5240, the most Fe-poor star known Christlieb et al. (2002).

${ }^{3} \mathrm{CD}-38^{\circ} 245$ is the most metal poor star known which is not extremely $\mathrm{C}$-enhanced. 
Table 3. Comparison of G77-61 with carbon-rich extremely metal poor stars.

\begin{tabular}{|c|c|c|c|c|c|c|c|}
\hline & \multirow{2}{*}{$\begin{array}{l}\text { G77-61 } \\
\text { X/Fe }]\end{array}$} & \multirow{2}{*}{$\begin{array}{l}\mathrm{CS} 29498-043 \\
{[\mathrm{X} / \mathrm{Fe}]^{a}}\end{array}$} & \multirow{2}{*}{$\begin{array}{l}\text { HE0107-5240 } \\
{[\mathrm{X} / \mathrm{Fe}]}\end{array}$} & \multicolumn{2}{|c|}{ CS22949-037 } & \multirow{2}{*}{$\begin{array}{l}\mathrm{CS} 22957-027 \\
{[\mathrm{X} / \mathrm{Fe}]^{a}}\end{array}$} & \multirow{2}{*}{$\begin{array}{l}\text { HE0007-1832 } \\
{[\mathrm{X} / \mathrm{Fe}]}\end{array}$} \\
\hline & & & & {$[\mathrm{X} / \mathrm{Fe}]$} & {$[\mathrm{X} / \mathrm{Fe}]^{a}$} & & \\
\hline $\mathrm{Li} \mathrm{I}$ & $<+4$ & & $<+5.3$ & & $<-0.3$ & & \\
\hline $\mathrm{C}(\mathrm{CH})$ & +2.6 & $+2.20^{b}$ & +3.70 & +1.28 & +1.27 & +2.4 & +2.55 \\
\hline${ }^{12} \mathrm{C} /{ }^{13} \mathrm{C}$ & 5 & $6^{c}$ & $>50$ & & 4 & 8 & 7 \\
\hline $\mathrm{N}(\mathrm{CN})$ & +2.6 & +2.26 & $+2.70^{a}$ & +2.84 & +2.66 & +1.62 & +1.85 \\
\hline $\mathrm{O}(\mathrm{CO})$ & & +2.45 & $+2.4^{d}$ & & +2.12 & & \\
\hline $\mathrm{Na} \mathrm{I}$ & +0.60 & +1.41 & +0.81 & & +2.09 & & \\
\hline Mg I & +0.49 & +1.74 & +0.15 & +1.21 & +1.35 & +0.65 & +0.76 \\
\hline $\mathrm{Ca} \mathrm{I}$ & +0.37 & +0.10 & -0.09 & +0.40 & +0.31 & +0.14 & +0.32 \\
\hline $\mathrm{Ca}$ II & +0.57 & & +0.36 & & & & \\
\hline Cr I & +0.36 & -0.41 & $<+0.26$ & -0.60 & -0.44 & & \\
\hline Mn I & -0.06 & -1.00 & $<+0.36$ & -0.97 & -0.84 & -0.41 & -0.23 \\
\hline $\mathrm{Fe} \mathrm{I}{ }^{e}$ & -4.03 & -3.49 & -5.28 & -3.74 & -3.94 & -3.12 & -2.65 \\
\hline $\mathrm{Zn} \mathrm{I}$ & $<2.7$ & $<+0.5$ & $<+2.65$ & & +0.63 & & \\
\hline Sr II & $<0$ & -0.57 & $<-0.52$ & -0.02 & +0.25 & -0.56 & +0.07 \\
\hline $\mathrm{Ba}$ II & $<1$ & -0.42 & $<+0.82$ & -0.89 & -0.61 & -1.23 & +0.16 \\
\hline $\mathrm{Eu}$ II & $<+3$ ? & & $<+2.78$ & $<+0.88$ & $<0.01$ & $<+0.83$ & $<+1.79$ \\
\hline Source & & $f$ & $g$ & $h$ & $i$ & $c$ & $j$ \\
\hline $\begin{array}{l}{ }^{a} \text { Correct } \\
{ }^{b} \text { From C } \\
{ }^{c} \text { Aoki et } \\
{ }^{d} \text { Bessell } \\
{ }^{e}[\mathrm{Fe} / \mathrm{H}] . \\
{ }^{f} \text { Aoki et } \\
{ }^{g} \text { Christli } \\
{ }^{h} \text { Norris } \\
{ }^{i} \text { Depagn } \\
{ }^{j} \text { Cohen }\end{array}$ & $\begin{array}{l}\text { for differe } \\
\text { (2002a). } \\
\text { al. (2004) } \\
(2004) \text {. } \\
\text { et al. (200 } \\
\text { 1. (2001). } \\
\text { al. }(2002 \\
\text { 1. }(2004) \text {. }\end{array}$ & $\mathrm{s}$ in solar referen & bundance. & & & & \\
\hline
\end{tabular}

lighter elements discussed further below. There are however discrepancies: $[\mathrm{Mn} / \mathrm{Fe}]$ is $\sim 0.5$ dex lower in the normal giants and dwarfs than it is in $\mathrm{G} 77-61$. [ $\mathrm{Cr} / \mathrm{Fe}]$ is more than 0.5 dex lower in the dwarfs, and 0.8 dex in the giants. The case of [K/Fe] almost 1 dex higher in G77-61 than in the mean giants, may be due to the difficulty of measuring the resonance $\mathrm{K}$ I lines affected by telluric absorption, and strongly blended with molecular lines. Despite this, the overall agreement between G77-61 and the mean metal-poor giants and dwarfs is remarkably good considering the difficulty of the analysis.

The abundance ratios in $\mathrm{C}$-rich metal poor stars, which obey the above relations for the elements between $\mathrm{Mg}$ and the Fe-peak, appear to float for the lighter and for the heavier elements beyond the Fe-Peak. Some extremely metal poor C-stars have large enhancements of the heavy elements, i.e. Ba, La and even $\mathrm{Pb}$ in some cases (see, e.g., Van Eck et al. 2003; Aoki et al. 2002c; Lucatello et al. 2003; Sivarani et al. 2004; Cohen et al. 2004). Those dwarfs with $s$-process enhancement must arise in binary systems with a more massive primary, now presumably a white dwarf, in which mass transfer has occured. Other very metal poor C-rich stars show no enhancement of the heavy elements (see, e.g. Aoki et al. 2002c; or Cohen et al. 2004). Although we only have rough upper limits to the abundance of elements heavier than the Fe peak in G77-61, it appears to be one of the latter group, with no or only modest enhancement of the heavy elements. Regarding mass-transfer, Wallerstein \& Knapp (1998) conjectured that the extremely low metallicity of G77-61 could be due to a loss of non-volatile elements during the mass transfer. This hypothesis does not seem supported by any observation. The metallicity now determined around $1 / 10000$ solar is not as extreme as previously believed, and the general pattern of abundances is close to other dwarfs and giants of similar metallicity. In addition, at such a high $\mathrm{C} / \mathrm{O}$ ratio the dust formed would be graphite or amorphous carbon, not incorporating $\mathrm{Mg}, \mathrm{Ca}, \mathrm{Fe}$, and other elements that condensate in e.g. silicates in O-rich environments.

With regard to the $\mathrm{CNO}$ elements, the general trend seen by the second author in examining the spectra of large numbers of very metal poor stars is that if $\mathrm{C}$ is highly enhanced, then $\mathrm{N}$ may be enhanced, but the range of enhancement varies significantly. On the other hand, $\mathrm{N}$ is never highly enhanced unless there is a substantial C-enhancement. We compare the deduced abundances for G77-61 with those of four very metal poor C-enhanced-stars in Table 3, the first of which is HE 0107-5240, the most Fe-poor star known, analyzed by Christlieb et al. (2004). There are two independent detailed abundance analyses of CS22949-037, a highly C-enhanced, extremely metal-poor giant, by Norris et al. (2001) and by Depagne et al. (2002). The agreement between them indicates the level of accuracy one can expect for stars with 
such complex spectra. However G77-61 is much cooler than this star, and its spectrum is correspondingly more complex. CS 22957-027 is another extremely metal poor giant with a very large C-enhancement studied by Norris et al. (1997) and by Aoki et al. (2002a); the results of the more recent analysis are shown in the table. CS 29498-043 (Aoki et al. 2002a,b, 2004 ) is a very metal poor giant with highly enhanced $\mathrm{Na}$ and $\mathrm{Mg}$ as well as CNO. HE 0007-1832 is a very metal poor dwarf with no enhancement of the heavy elements (Cohen et al. 2004).

Table 3 gives an indication of the variety found among the C-rich metal poor stars. The four stars listed have $N(\mathrm{C}) / N(\mathrm{~N})$ ranging over more than a factor of 1000 , with no obvious correlation with $[\mathrm{Fe} / \mathrm{H}]$. G77-61 and all of the comparison stars, except HE0107-5240, though presumably a giant (Christlieb et al. 2004 ), show low $\mathrm{C}^{12} / \mathrm{C}^{13}$ ratios, indicating substantial $\mathrm{CN}$ processing. If one ignores the $\mathrm{CNO}$ elements, a crucial distinction among them involves the elements just heavier than CNO, the easiest of which to detect are $\mathrm{Na}$ and $\mathrm{Mg}$. If those elements are not enhanced, then the abnormality is confined to the enhancement of the CNO elements, and an origin involving normal processes of nucleosynthesis within stars of intermediate mass can be imagined; for example a binary scenario with no heavy element production is viable. However, if $\mathrm{Na}$ and $\mathrm{Mg}$ are enhanced, as is the case especially for CS 22949-037 and for CS 29498-043, and to a lesser extent for the other three stars, then conventional wisdom suggests that significant production of $\mathrm{Mg}$ and $\mathrm{Na}$ is not expected for intermediate mass metal-poor stars, and a supernova event is required. It is these arguments that have led to the discussion of SN with fallback models for extremely metal poor massive stars Umeda \& Nomoto (2003) and Tsujimoto \& Shigeyama (2003) where most of the lighter elements, produced in the outer zones, get ejected, but the mass cut is such that the heavier elements fall back onto the collapsed core. The abundance ratios of the ejecta can be fine-tuned by invoking mixing as well as fallback.

However, very recent calculations by Herwig (2005) and by Karakas (2005) demonstrate that intermediate mass very low metallicity AGB stars can produce and mix to their surface substantial amounts of $\mathrm{Na}$ and $\mathrm{Mg}$, as well as copious amounts of $\mathrm{C}$ and $\mathrm{N}$, and a low ${ }^{12} \mathrm{C} /{ }^{13} \mathrm{C}$ ratio due to $\mathrm{CN}$ processing. This eliminates the need to seek a type II SN origin for their excesses in G77-61 and in the other stars listed in Table 3. In addition, according to Goriely \& Siess (2004), the s-process efficiency may be very much reduced in low metallicity AGB stars, due to the higher temperature of the bottom of the convective zone. This could explain the class of very metal-poor carbonrich stars without excess of heavy elements to which G77-61 seems to belong.

We may thus view the $\mathrm{O}$ abundance as the key to separating excesses of the light elements $\mathrm{C}, \mathrm{N}, \mathrm{Na}, \mathrm{Mg}$ as arising from type II SN of some kind versus binary mass transfer involving an extremely low metallicity AGB star. In the former case, copious $O$ should be present, while in the latter, O should not be significantly enhanced. HE0107-5240 falls into the former category, as do at least two of the comparison stars. But preliminary information regarding the $\mathrm{O}$ abundance in G77-61 based on the low-resolution IR spectra of Joyce (1998) is that the $\mathrm{O}$ abundance is low. However, Joyce ascribed the apparent weak CO both to the low metallicity of G77-61 and at least partially to suppression of near-IR molecular bands by collisioninduced absorption $\left(\mathrm{H}_{2}-\mathrm{H}_{2}\right.$, and to a lesser extend $\left.\mathrm{He}-\mathrm{H}_{2}\right)$. Our model atmosphere calculations with high $\mathrm{O}$ abundance, and including collision-induced absorption predict strong $\mathrm{CO}$ bands. In any case all $\mathrm{SN}$ models tend to produce large amounts of $\mathrm{O}$, relative to $\mathrm{C}$, and it is therefore crucial to determine the $\mathrm{O}$ abundance in G77-61 to separate the binary AGB from the SN hypothesis for the selective abundance enhancements in this extremely metal-poor dwarf carbon star. A high resolution near-IR spectrum is already in hand and results should be available in the near future.

Acknowledgements. We thank N. Christlieb for suggesting a reinvestigation of this star. B. Freytag is thanked for suggesting the microturbulence estimate, and R. Cayrel and E. josselin for useful comments. The entire Keck/HIRES user communities owes a huge debt to Jerry Nelson, Gerry Smith, Steve Vogt, and many other people who have worked to make the Keck Telescope and HIRES a reality and to operate and maintain the Keck Observatory. We are grateful to the W. M. Keck Foundation for the vision to fund the construction of the W. M. Keck Observatory. The authors wish to extend special thanks to those of Hawaiian ancestry on whose sacred mountain we are privileged to be guests. Without their generous hospitality, none of the observations presented herein would have been possible. J.G.C. is grateful to the National Science Foundation for partial support under grant AST-0205951. This publication makes use of data from the Two Micron All-Sky Survey, which is a joint project of the University of Massachusetts and the Infrared Processing and Analysis Center, funded by the National Aeronautics and Space Administration and the National Science Foundation.

\section{References}

Allende-Prieto, C., Lambert, D. L., \& Asplund, M. 2002, ApJ, 573, L137

Alvarez, R., \& Plez, B. 1998, A\&A, 330, 1109

Aoki, W., Norris, J. E., Ryan, S. G., Beers, T. C., \& Ando, H. 2002a, ApJ, 576, L141

Aoki, W., Norris, J. E., Ryan, S. G., Beers, T. C., \& Ando, H. 2002b, PASJ, 54, 933

Aoki, W., Ryan, S. G., Norris, J. E., et al. 2002c, ApJ, 580, 1149

Aoki, W., Norris, J. E., Ryan, S. G., et al. 2004, ApJ, 608, 971

Asplund, M. 2003, in CNO in the Universe, ed. G. Charbonnel, D. Schaerer, \& G. Meynet, ASP Conf. Ser., 304, 275

Asplund, M., Gustafsson, B., Kiselman, D., \& Eriksson, K. 1997, A\&A, 318, 521

Asplund, M., Nordlund, A., Trampendach, R., \& Stein, R. 2000, A\&A, 359, 743

Asplund, M., Grevesse, N., Sauval, A. J., Allende Prieto, C., \& Kiselman, D. 2004, A\&A, 417, 751

Bessell, M. S., \& Brett, J. M. 1988, PASP, 100, 1134

Bessell, M. S., Christlieb, N., \& Gustafsson, B. 2004, ApJ, 612, L61

Carpenter, J. M. 2001, AJ, 121, 2851

Cayrel, R., Depagne, E., Spite, M., et al. 2004, A\&A, 416, 1117

Christlieb, N., Bessell, M. S., Beers, T. C., et al. 2002, Nature, 419, 904

Christlieb, N., Gustafsson, B., Korn, A. J., et al. 2004, ApJ, 603, 708

Cohen, J., Christlieb, N., McWilliam , A., et al. 2004, ApJ, in press

Cooper, N. 1975, JQSRT, 15, 139 
Cutri, R. M., et al. 2003, Examplanatory Supplement to the 2MASS All-Sky Data Release, http://www.ipac.caltech.edu/ 2mass/releases/allsky/doc/explsup.html

Dahn, C. C., Liebert, J., Kron, R. G., Spinrad, H., \& Hintzen, P. M. 1977, ApJ, 216, 757

Dearborn, D. S. P., Liebert, J., Aaronson, M., et al. 1986, ApJ, 300, 314

Depagne, E., Hill, V., Spite, M., et al. 2002, A\&A, 390, 187

Edvardsson, B., Andersen, J., Gustafsson, B., et al. 1993, A\&A, 275, 101

Erman, P., \& Iwamae, A. 1995, ApJ, 450, L31

Gass, H., Liebert, J., \& Wehrse, R. 1988, A\&A, 189, 194

Goriely, S., \& Siess, L. 2004 A\&A, 421, L25

Grevesse, N., \& Sauval, J. 1998, Space Sci. Rev., 85, 161

Grevesse, N., Lambert, D. L., Sauval, A. J., et al. 1991, A\&A, 242, 488

Gustafsson, B., Bell, R. A., Eriksson, K., \& Nordlund, Å. 1975, A\&A, 42,407

Gustafsson, B., Edvardsson, B., Eriksson, K., et al. 2003, in Stellar Atmosphere Modeling, ASP Conf. Ser., 288, 331

Harris, H. C., Dahn, C. C., Walker, R. L., et al. 1998, ApJ, 502, 437

Harris, G. J., Lynas-Gray, A. E., Miller, S., \& Tennyson, J. 2004, ApJ, 600,1025

Herwig, F. 2005, ApJS, 155, 651

Hill, V., Plez, B., Cayrel, R., et al. 2002, A\&A, 387, 560

Houdashelt, M. L., Bell, R. A., \& Sweigart, A. V. 2000, AJ, 119, 1448

Joyce, R. R. 1998, AJ, 115, 2059

Karakas, A. I. 2005, Ph.D. Thesis, Monash University
Kim, Y.-C., Demarque, P., Yi, S. K., \& Alexander, D. R. 2002, ApJS, 143, 499

Kupka, F., Piskunov, N. E., Ryabchikova, T. A., Stempels, H. C., \& Weiss, W. W. 1999, A\&AS, 138, 119

Langhoff, S. R., Bauschlicher, C. W., Jr., Rendell, A. P., \& Komornicki, A. 1990, JCP, 92, 3000

Lucatello, S., Gratton, R., Cohen, J. G., et al. 2003, AJ, 125, 875

Neale, L., \& Tennyson, J. 1995, ApJ, 454, L169

Norris, J. E., Ryan, S. G., \& Beers, T. C. 1997, ApJ, 489, L169

Norris, J. E., Ryan, S. G., \& Beers, T. C. 2001, ApJ, 561, 1034

Plez, B., Brett, J. M., \& Nordlund, Å. 1992, A\&A, 256, 551

Querci, F., Querci, M., \& Kunde, V. G. 1971, A\&A, 15, 256

Richter, J., \& Tonner, K. F. 1967, Z. Astrophys., 67, 155

Routly, P. M. 1972, Pub. US Naval Obs., 20, part 6

Schlegel, D. J., Finkbeiner, D. P., \& Davis, M. 1998, ApJ, 500, 525

Sivarani, T., Bonifacio, P., Molaro, P., et al. 2004, A\&A, 413, 1073

Shortridge, K. 1993, in Astronomical Data Analysis Software and Systems II, ed. R. J. Hannisch, R. J. V. Brissenden, \& J. Barnes, A.S.P. Conf. Ser., 52, 219

Skrutskie, M. F., Schneider, S. E., Stiening, R., et al. 1997, in The Impact of Large Scale Near-IR Sky Surveys, ed. F. Garzon et al. (Dordrecht: Kluwer), 187

Van Eck, S., Jorissen, A., Goriely, S., \& Plez, B. 2003, A\&A, 404, 291

Tsujimoto, T., \& Shigeyama, T. 2003, ApJ, 584, L87

Umeda, H., \& Nomoto, K. 2003, Nature, 422, 871

Vogt, S. E., Allen, S. L., Bigelou, B. C., et al. 1994, SPIE, 2198, 362

Wallerstein, G., \& Knapp, G. R. 1998, ARA\&A, 36, 369

Yi, S. K., Kim, Y.-C., \& Demarque, P. 2003, ApJS, 144, 259 